\title{
Musculoskeletal Deformity, CTCAE
}

National Cancer Institute

\section{Source}

National Cancer Institute. Musculoskeletal Deformity, CT CAE. NCI Thesaurus. Code C143687.

A disorder characterized by a malformation of the musculoskeletal system. 